\title{
Difficulties in the Measurement of Pulse-Wave Velocity
}

\author{
W. Klip, M.D., Ph.D., Utrecht, Netherlands
}

\section{INTRODUCTION}

The purpose of this paper is to discuss the difficulties encountered in the measurement of pulse-wave velocity. The methods themselves will not be discussed in detail, because they are not pertinent to this paper.

Since the pulse-wave velocity is greater in rigid tubes, a determination of this velocity could be used to give a quantitative estimate as to arterial wall rigidity, either secondary to arteriosclerosis or to age changes.

\section{DIFFICULTIES WITH INSTRUMENT REGISTRATION}

The accurate measurement of pulse-wave velocity is not a simple matter, as can be demonstrated with the help of an old-fashioned instrument, the Marey tambour. To illustrate, take two tambours and place them so that their pointers rest parallel on a kymugraph revolving at a speed of $2.5 \mathrm{~cm}$. per second, in the manner illustrated below. Imagine that the sensing diaphragms are placed on the radial and brachial arteries, respectively, and that the distance between the diaphragms, $s$, is known. Then the pulse-wave velocity can be computed using the formula

$$
v=s / t
$$

where $\mathrm{s}=$ distance and $\mathrm{t}=$ time in seconds (Figs. 1 and 2). However, the actual measurement is not so simple, because, for one thing, the tambour is an instrument of great inherent inertia, and thus cannot follow the more rapid pressure variations. Modern registration methods are also plagued with similar difficulties, which, however, find their origin in the tissues. For further insight, let us take a closer look at the tambour.

1. Amplitude Characteristics.-Suppose we strike the tambour. The pointer will vibrate with a resonant frequency $\left(\nu_{1}\right)$ that is independent of the applied force, and depends only on the mechanical properties of the tambour, i.e., the length of the pointer arm, et al. Now let us apply artificially a periodic pressure variation of constant amplitude to the tambour, so that it moves in simple harmonic motion, with a given frequency which is small at first, but which is gradually

From the Department of Medical Physics, Physical Laboratory of the University of Utrecht, Netherlands (Prof. Dr. H. C. Burger, Head of the Department), and from the Netherlands Organization for Applied Scientific Research (T.N.O.).

Received for publication July 21, 1958. 
increased. We must choose the measured pressure variations small so that the needle excursions are linear with respect to the pressure. Thus, we see the recording of Fig. 3 on the drum as the frequency is increased.

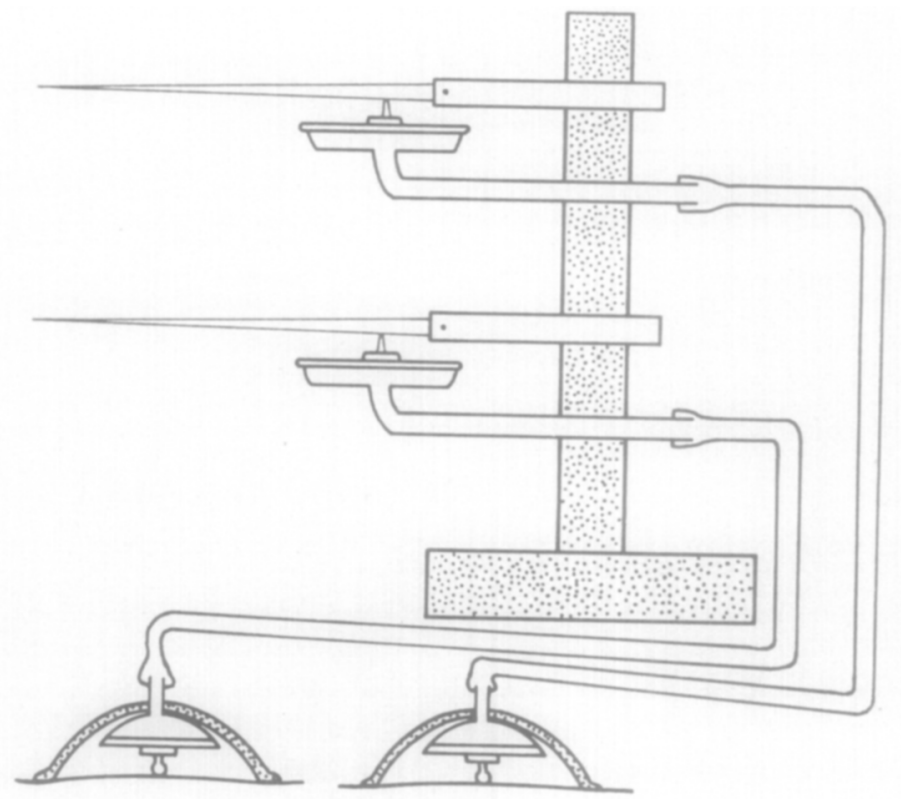

Fig. 1.-Two tambours.

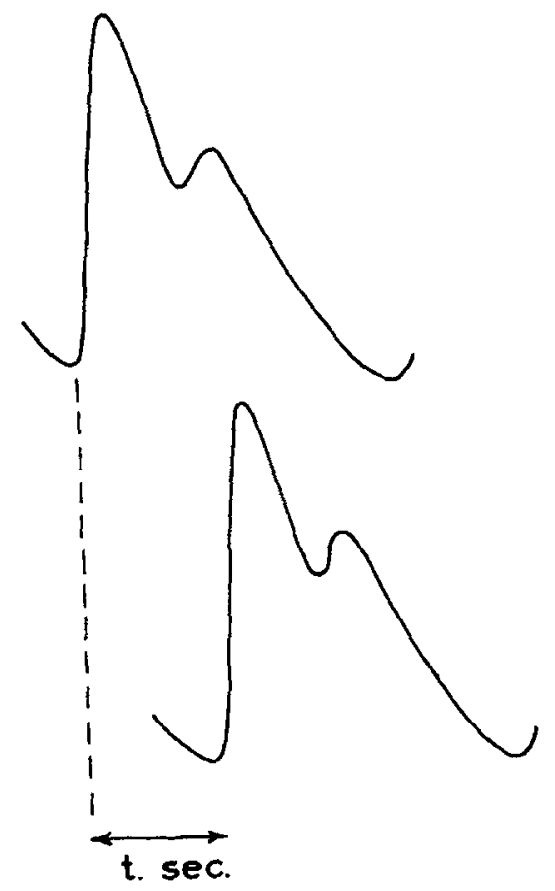

Fig. 2.-Two ideal pulse curves. 
At first the needle gives a true recording of the pressure, but as the resonant frequency of the recording system is reached, the excursions become greater in spite of the fact that the pressure variation has remained constant throughout the recording time. Referring again to Fig. 3, we see that if the frequency is further increased, the inertia drag of the tambour becomes excessive and the needle movement ineffective. Therefore, it is apparent that the pressure is correctly recorded only for frequencies appreciably lower than the resonant frequency of the recording system. The height of the maximum in Fig. 3 depends on the damping.

Let us re-examine the pressure curve. A phenomenon which repeats itself with a period $\mathrm{T}_{2}=\frac{1}{\nu_{2}}$ (e.g., $\mathrm{T}_{2}= \pm 1$ sec.) (Fig. $4, a$ ) can be considered as the sum of an infinite number of harmonic

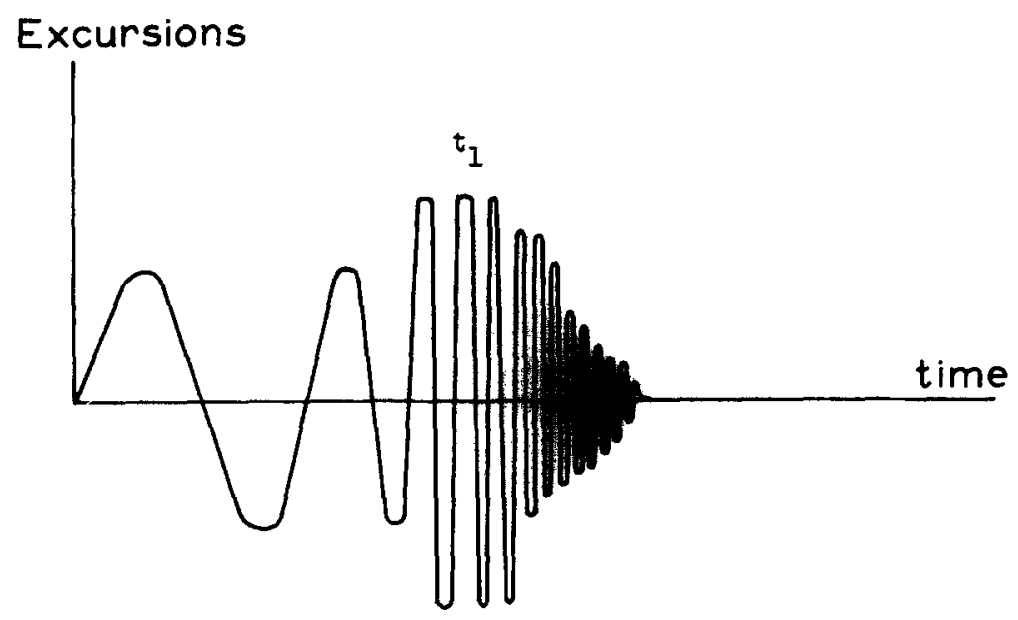

Fig. 3.-Frequency increases with time. At $t_{1}$, a frequency $\nu_{1}$ is passed.

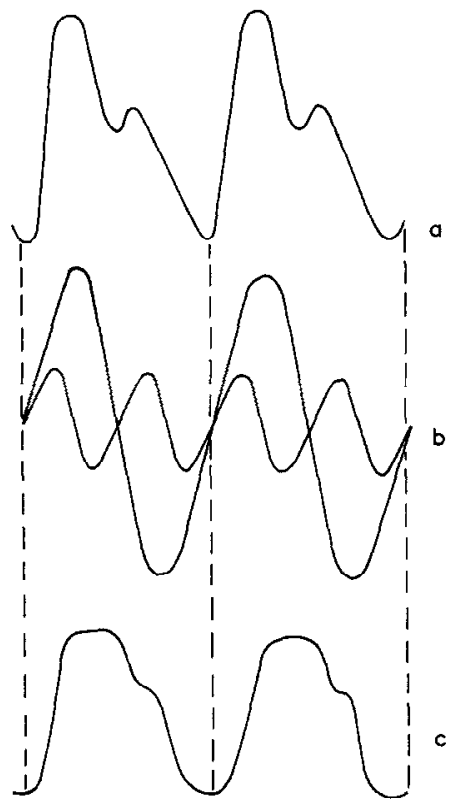

Fig. 4.- $a$, Pulse curve, $b$, Fourier analysis (grand frequency and first harmonic). $c$, Distorted pulse contour. 
vibrations with frequencies of $\nu_{2}, 2 \nu_{2}, 3 \nu_{2}$ ad infinitum, each with different amplitude and phase (Fourier analysis, Fig. $4, b$ ). In the normal pulse wave, the harmonics which have a frequency greater than 30 cycles per second also have a very low amplitude and are of no practical importance. The tambour magnifies harmonics with a frequency of 3 cycles per second too much if its resonant frequency $\nu_{1}=3$, thereby giving a distorted pressure curve; for example, it gives Fig. $4, c$ instead of Fig. $4, a$.

Such a distortion can result in a tremendous error in the determination of pulse-wave velocity by simply shifting the foot-point in the distorted curve. It is only by choosing two exactly identical registering systems that this difficulty can be eliminated.

2. Phase Characteristics. - It is also true that the tambour will give each harmonic a different shift in the horizontal direction, thereby giving rise to further distortion in the recorded pulse (see Fig. 4).

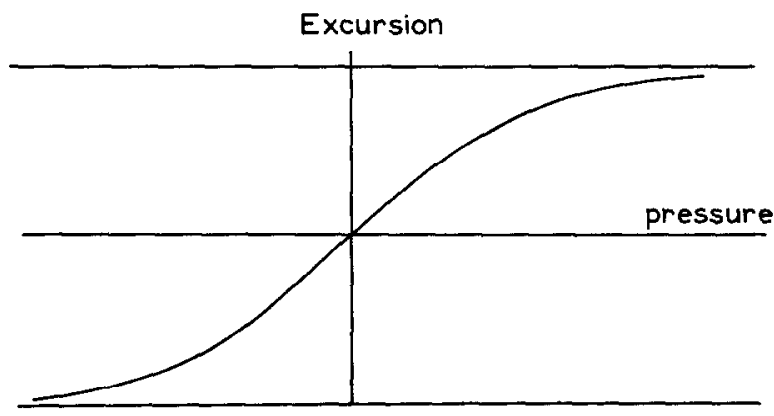

Fig. 5.--The phenomenon of alinearity.

3. Nonlinearity.-If amplitude characteristics are to be studied, it is necessary that small pressure variations be chosen, for it is only in the low-pressure range (Fig. 5) that the excursion is proportional to the pressure. Large pressure accretions would be recorded with gross error.

\section{EXTRANEOUS TISSUE INTERFERENCE}

Difficulties inherent within the recording instrument can be reduced by using more modern instruments which have resonant frequencies greater than 30 cycles per second and adjusted damping. The utilization of such instruments will allow equal magnification of all amplitudes of the different harmonics of the pulse wave, thereby giving a negligible phase shift and also fulfilling the requirements of linearity.

Even with the instrumentation corrected, more difficulties arise. Between the blood, the propagating medium, and the sensing device there are extraneous tissues, namely, the vessel wall and subcutaneous and cutaneous tissues. These tissues function just as a tambour, with all its inherent bad qualities, as discussed above (amplitude distortion, phase shift, and nonlinearity). So, no matter how good our instrument is, the pressure wave will still be registered in a distorted form.

The above-mentioned problem would make little practical difference if the deformation were constant at all registration sites, but tissue characteristics above the lumen of the radial artery differ from, say, lissue characteristics above the brachial artery, etc. 
Furthermore, the pressure variations recorded at central sites are greater than those recorded peripherally. Thus, according to Fig. 5, the former may lie in the nonlinear part of the curve (vessel-wall distortion).

\section{THE COURSE OF THE VESSEL}

We can largely abolish the deformation by measuring the pressure directly with a needle connected to the proper electronic recording device, but the disadvantage of performing a surgical procedure is obvious. Another method which might be tried involves the use of a small, blunt piston with a stiff spring which may be pressed into the skin and vessel wall, thereby obtaining a displacement of the vessel wall proportional to the pressure change. The vessel wall, subcutaneous and cutaneous tissue, and the stamp, form in this case a system with a resonant frequency far above 30 cycles per second. Also, because of the large stiffness factor ("restoring force") of the springs, the excursions of the stamp are small, so that linearity exists. Even if this succeeds, the pressure curves measured centrally differ from those measured peripherally. This deformation cannot be eliminated, because this distortion occurs as the wave travels from central to peripheral segment (e.g., brachial to radial segment). It appears that each part of the vessel passed by the wave forms with the surrounding tissues a vibrating system (just like the tambour) with resonant frequencies less than 30 cycles per second. Further deformation results from wave reflection against arterial branches and against the "periphery." Thus, if we assume that the maximum points of two curves measured along an arterial segment are "equal," the velocity computed from the measured distance is not at all a good approximation to the "true velocity." In all cases we find two different results when maxima and foot-points are used respectively.

\section{INFLUENCE OF FREQUENCY}

Even if we could assume that the vessel wall and surrounding tissues were constant, deformation would still result from the fact that different harmonics of the pulse wave are propagated with a different velocity. This gives a phase shift, and the end result is a deformed peripheral curve.

\section{ALINEARITY OF DIFFERENTIAL EQUATIONS}

Suppose that we made the pressure at the first part of a straight, isotropic (homogeneous) tube vibrate in simple harmonic motion, so that there was only one frequency. Still, deformation would occur, but not deformation in the strictest sense of the word. The cause of the "deformation" here is due to the fact that, on theoretical grounds, the pulse wave does not exist! The wave would exist if certain terms in the differential equations of motion could be neglected, i.e., the products of displacements of particles in the fluid, the vessel walls and their derivatives with respect to the displacements themselves. If this neglect is allowed, the approximation may be too rough, but it is only in this way that a solution of the differential equation can be derived having the form of a phe- 
nomenon which has a velocity of propagation, i.e., $P=f(x-c t)$. For, from

$$
f\left(x_{0}-c t\right)=f\left\{x_{1}-c\left(t+\frac{x_{1}-x_{0}}{c}\right)\right\}=f\left\{x_{1}-c(t+\tau)\right\}
$$

we see that the pressure in a point $x_{0}$ at a time, $t$, will be the same $\tau=\frac{x_{1}-x_{0}}{c}$ seconds later at some other point, $\mathrm{x}_{1}$, so that $c=\frac{x_{1}-x_{0}}{\tau}$ can be termed the velocity of propagation of the pressure. Thus, there is pressure-wave propagation if we are allowed to neglect the terms mentioned above. Without neglecting these terms, the solution of the differential equation has the general form $P=f(x, t)$, i.e., the two independent variables $x$ and $t$ cannot now be unified into one single, independent variable, $Y$, defined by $Y=x-c t$, and so a velocity of propagation need not exist, for it is unlikely that a number, $c$, can be found so that at every point $x_{1}$, the relation

$$
f\left(x_{0}, t\right)=f\left\{x_{1}, t+\frac{x_{1}-x_{0}}{c}\right\}
$$

is fulfilled. If we plot $f(x-c t)$ at two different times as a function of $x$, we get two identical curves. They are only displaced with respect to each other along the axis of $x$. However, if we plot $f(x, t)$ at two different times, we get two curves that may differ totally.

Hence, on theoretical grounds there is no true pulse-wave propagation, and we are forced, therefore, to define the pulse-wave velocity empirically. We must, for example, say that the two foot-points (Fig. 2) are by definition corresponding pcints, and that the velocity is equal to the distance, $x_{2}-x_{1}$, divided by the time, $t_{2}-t_{1}$. There is little wonder that when two other points are considered, totally different "velocity" values are obtained, even negative values in some cases.

\section{DIFFERENT MODES OF VIBRATION}

Suppose we consider another problem: In considering tubes in which the displacements are small and their products are therefore negligible, there is still deformation. To demonstrate: If a simple harmonic oscillation is transmitted through an isotropic tube, longitudinal, torsional, and flexural waves can all be produced, each with its own velocity or velocities. It is thus probable that two wave forms exist simultaneously, and because of their velocity difference these waves will shift in relation to each other as they course through the tube. The end result is a wave exhibiting an entirely different form, i.e., deformation.

\section{EXPERIMENTS}

Since we know that something like a pulse wave exists, in spite of the absence of a theoretical basis, there must be, roughly speaking, a self-propagating phenomenon. So, the prerogative of neglecting certain terms in the differential equations is not totally absurd. Experimentally, the pulse-wave velocity has been measured by many investigators, but the results are not hopeful. The spreading of values in normal persons is so great that if in an arteriosclerotic person a large velocity is recorded, one may not conclude that wall rigidity was 
the cause. Spreading may originate from any of three causes: (1) measuring apparatus, (2) deformation, as discussed above, and (3) condition of the person, such as meals (see Table II) and respiration. The velocity on one day may be \pm 20 per cent greater on the next day in the same person. The measuring apparatus and the condition of the person can certainly be improved upon, but little if any improvement can be expected in regard to deformation.

It is still possible to conceive that determinations of pulse-wave velocity may be performed at some time in the same individual so as to study the influence of therapeutic measures. It is also quite possible that the study of certain wave characteristics may have clinical importance.

The uncertainty raised by the possibility of deformation of the pulse wave can be seen in the tables below.

A. Deformation of the Curve-We see from Table I that: (1) The velocity in the old man is significantly greater than that in the boy. (2) The velocity in the boy did not differ in the two arterial segments, but in the old man the velocity in the brachium was twice that in the antebrachium. Calcification could be responsible but deformation is also a likely cause.

TABLE I

\begin{tabular}{|c|c|c|}
\hline & BRACHIUM & ANTEBRACHIUM \\
\hline SUBJECT'S AGE & $\begin{array}{l}\text { VELOCITY AND STANDARD ERROR } \\
(\mathrm{CM}, / \mathrm{SEC} .)\end{array}$ & $\begin{array}{l}\text { VELOCITY AND STANDARD ERROR } \\
(\mathrm{CM} . / \mathrm{SEC} .)\end{array}$ \\
\hline $\begin{array}{l}11 \text { years } \\
80 \text { years }\end{array}$ & $\begin{array}{r}955 \pm 35 \\
2,470 \pm 200\end{array}$ & $\begin{array}{r}895 \pm 87 \\
1,330 \pm 62\end{array}$ \\
\hline Quotient & 2.6 & 1.5 \\
\hline
\end{tabular}

Fifty measurements were made in each subject.

\section{B. Condition of the Patient.-}

Meals: In Table II the influence of meals is recorded in an 11-year-old boy. From the table it is noted that after a meal there is a significant change in pulsewave velocity in peripheral vessels. This is not true for the aorta. The reason for this is not known but one can theorize that immediately after a meal there is peripheral vasoconstriction with vasodilatation in the splanchnic area. From

TABLE II

\begin{tabular}{c|c|c|c|c|c|c|c}
\hline \multicolumn{2}{c|}{ AORTA } & \multicolumn{2}{|c|}{ LEG } & \multicolumn{2}{|c|}{ BRACHIUM } & ANTEBRACHIUM \\
\hline $\begin{array}{c}\text { WITHOUT } \\
\text { MEALS }\end{array}$ & $\begin{array}{c}\text { WITH } \\
\text { MEALS }\end{array}$ & $\begin{array}{c}\text { WITHOUT } \\
\text { MEALS }\end{array}$ & $\begin{array}{c}\text { WITH } \\
\text { MEALS }\end{array}$ & $\begin{array}{c}\text { WITHOUT } \\
\text { MEALS }\end{array}$ & $\begin{array}{c}\text { WITH } \\
\text { MEALS }\end{array}$ & $\begin{array}{c}\text { WITHOUT } \\
\text { MEALS }\end{array}$ & $\begin{array}{c}\text { WITH } \\
\text { MEALS }\end{array}$ \\
\hline $436 \pm 13$ & $423 \pm 8$ & $806 \pm 10$ & $1,028 \pm 18$ & $955 \pm 23$ & $1,460 \pm 46$ & $890 \pm 87$ & $1,111 \pm 42$
\end{tabular}


Young's classic formula

$$
V e l=\frac{D E}{2 \rho_{r}}
$$

where $\mathrm{D}=$ wall thickness, $\mathrm{E}=$ Young's modulus, $\mathrm{r}=$ radius of the tube, $\rho=$ density of the blood, one "can see that an arterial constriction could affect the wave velocity ( $D$ and $E$ supposed constant).

Respiralion: If inspiration is held for about half a minute, quite often there is an increase of 30 per cent or more in the pulse-wave velocity. The respiratory influence is especially manifest in peripheral vessels. Because the effect of a meal and respiration is smaller in the aorta, that vessel is perhaps preferable for measurements of the pulse-wave velocity.

SUMMARY

The methods employed in the measuring of pulse-wave velocity are subject to gross error because of alinearity and frequency distortion resulting from lowresonance frequencies of instrumentation and arterial segment and extraneous tissue interference. Deformation also occurs because of different speeds of propagation of the various pulse harmonics.

Some examples are presented showing the effect of meals on the pulse-wave velocity. Respiration also has a great influence.

The author wishes to thank Carl J. Bentzel for the translation and advice. 